\title{
AN APPROACH TO QUASILINEAR ELLIPTIC PROBLEMS
}

\author{
BY A. E. HURD AND C. F. SCHUBERT ${ }^{1}$ \\ Communicated by A. E. Taylor, April 23, 1964
}

1. Introduction. Some of the common methods used to prove existence theorems for quasilinear elliptic equations employ the Schauder fixed-point theorem, the Schauder-Leray theory, or some variant of these in a Banach space [1], [3], [4]. It is also usual to consider the principal part of the equation to be in divergence form, when strong ellipticity and the Dirichlet problem are natural concepts [1], [5], [11]. This leaves the question of regularity of the solution to be dealt with separately. With the wealth of existence and regularity theory now available for general linear elliptic equations it seems desirable to extend this work as directly as possible to quasilinear elliptic equations with continuous coefficients but without assuming divergence form. This announcement indicates some results on this approach. In all applications we rely on known linear existence and regularity theory. Unavoidably the technique requires the existence of an a priori estimate, but very naive estimates can be made to yield results easily. The results so obtained are usually not the most general ones known in specific cases. The central ideas are presented in the next section as two theorems which can be both generalized and specialized considerably as abstract theorems. The form we have given however, is that most useful in applications to quasilinear elliptic equations. Some simple examples of the use of these theorems are given in the last section. Proofs and the application to general elliptic and parabolic equations will appear elsewhere.

2. Existence theorems. Let $X, Y$ be Banach spaces with norms denoted by $|\cdot|$, in both spaces, and denote by $[X, Y]$ the Banach space of continuous linear operators on $X$ into $Y$ with the uniform topology. Let $\theta: u \rightarrow \theta(u)$ be a not necessarily linear mapping from $X$ into $[X, Y]$ with the following properties: there exists a closed convex subset $U$ of $X$ and a subset $W$ of $Y$ such that

(2.1) the restriction of $\theta$ to $U$ is compact, i.e., $\theta$ maps a bounded sequence of elements of $U$ into a sequence of elements of $[X, Y]$ containing a convergent subsequence,

(2.2) the restriction of $\theta$ to $U$ is continuous, i.e., if $\left\{u_{n}\right\}$ is a sequence of elements of $U$, such that $u_{n} \rightarrow u \in U$ as $n \rightarrow \infty$ then $\theta\left(u_{n}\right) \rightarrow \theta(u)$ in $[X, Y]$ as $n \rightarrow \infty$,

1 The research and preparation of this paper was supported (in part) by the National Science Foundation grants GP-2045 and GP-1988. 
(2.3) for each $u \in U$, the range of the restriction of $\theta(u)$ to $U$ contains $W$, and $\theta(u)$ has an inverse defined on all of $W$.

Definition 2.1. If $\theta$ satisfies (2.1)-(2.3), call $u \rightarrow \theta(u) u$ a quasilinear map from $U$ to $W$.

If $U=X, W=Y$ and $u \rightarrow \theta(u) u$ is a quasilinear map from $X$ to $Y$, the closed graph theorem guarantees that for each $u \in X$, there exists a $k(u)>0$ such that $|\theta(u) v| \geqq k(u)|v|$ for all $v \in X$. In the case of general $U, W$, we assume that for some fixed $\alpha \geqq 0$, the function $s \rightarrow k(s)$ defined by (2.4) (following) is positive for at least some interval of $s$ values. Here

$$
k(s)=\inf _{|u| \leqq s} \inf \{|\theta(u) v|+\alpha\}|v|^{-1},
$$

where the infima are taken over the indicated $u, v \in U$. Thus we assume for $\theta$ an a priori estimate of the form $|\theta(u) v|+\alpha \geqq k(s)|v|$ for all $u, v \in U$ with $|u| \leqq s$.

Using the Schauder fixed-point theorem in the form that a completely continuous, i.e., compact and continuous, map of a closed bounded convex subset of $X$ into itself has a fixed point [2], [3], we have the following theorem.

TheOREM 2.1. Suppose $u \rightarrow \theta(u) u$ is a quasilinear map from $U$ to $W$, and $f \in W$. If for some $s_{0}$, we have $|f| \leqq s_{0} k\left(s_{0}\right)$, then there exists a $u \in U$, with $|u| \leqq s_{0}$, such that $\theta(u) u=f$.

Let $B: u \rightarrow B u$ be a mapping, not necessarily linear, from $X$ into $Y$, such that

$$
\begin{gathered}
U \subset \text { domain of } B, \quad \text { range of } B \subset W, \\
B \text { is completely continuous on } U .
\end{gathered}
$$

If $W$ has the additional property

$$
\text { if } w_{1}, w_{2} \in W, \quad \text { then } w_{1}+w_{2} \in W,
$$

then certain operators, $B$, satisfying (2.5) and (2.6), can be regarded as perturbations of $\theta(u) u$ depending on the behaviour of $\phi(s)$ defined by

(2.8) $\phi(s)=\sup |B u|$, where the supremum is taken over $u \in U$, with $|u| \leqq s$.

This result is contained in the next theorem.

TheOREM 2.2. Suppose $u \rightarrow \theta(u) u$ is a quasilinear map from $U$ to $W$, $W$ satisfies (2.7), and $B$ is an operator satisfying (2.5) and (2.6). Then 
if $f \in W$, and for some $s_{0}$ we have $|f|+\alpha \leqq s_{0} k\left(s_{0}\right)-\phi\left(s_{0}\right)$, there exists a $u \in U$, with $|u| \leqq s_{0}$, such that $\theta(u) u-B u=f$.

3. Examples. Since the method is applicable equally to $L^{p}$ and Hölder estimates we give examples of the use of both. Let $G$ be a bounded domain in $E^{n}$. In the first two examples we assume that the boundary $\partial G$ of $G$ is of class $C^{4}$. For functions $u \in C^{\infty}(\bar{G})$ and $p>n$ we use the norm

$$
\|u\|_{j, p}=\left\{\sum_{|\mu| \leq j} \int_{G}\left|D^{\mu} u\right|^{p} d x\right\}^{1 / p}
$$

where the summation is over all derivatives of order $\leqq j$. Let $H^{j, p}(G)$ denote the completion of $C^{\infty}(\bar{G})$ with respect to this norm. Let $\phi \in C^{2}(\partial G)$ and use on $\phi$ the boundary norm $\langle\phi\rangle_{2-(1 / p), p}$ defined in [10]; this norm can be extended to a function in $C^{2}(\partial G)$. Let

$$
\begin{aligned}
U & =\left\{u \in H^{2, p}(G) ; u=\phi \text { on } \partial G\right\}, \\
W & =\left\{w \in L^{p}(G) ; w \in C(\bar{G})\right\} .
\end{aligned}
$$

Example 1. With $U, W$ as in (3.2) and $\theta(u)=\Delta=\sum_{i} \partial^{2} / \partial x_{i}^{2}$, application of Theorem 2.2 gives

THEOREM 3.1. Let $f(x, u, \partial u / \partial x)$ be a continuous function of its arguments for all $x \in \bar{G}$. Suppose that there exists a constant $C>0$, and a function $\eta(t)$, with $\lim _{t \rightarrow \infty} \eta(t)=0$, such that for each $u \in U$, $|f(x, u, \partial u / \partial x)| \leqq C+\eta\left(t_{u}\right) t_{u}$, where $t_{u}=\max _{x \in \bar{G}}\left\{|u|+\sum_{i}\left|\partial u / \partial x_{i}\right|\right\}$. The problem $\Delta u=f(x, u, \partial u / \partial x)$, in $G, u=\phi$ on $\partial G$, then has a solution $u \in C^{2}(G)$.

REMARK 1. This theorem allows more general perturbations of $\Delta$ than those of [6], [7], [11], since it permits $\partial u / \partial x$ on the right. The conclusion of the theorem is valid if the Dirichlet boundary condition is replaced by $\partial u / \partial n+a(x) u=\phi$ on $\partial G$, where $a(x) \in C^{2}(\partial G), a(x) \geqq 0$, and is strictly positive somewhere on $\partial G$.

Restricting $U, W$ further, e.g.,

$$
\begin{aligned}
U & =\left\{u \in H^{2, p}(G) ; u=\phi \text { on } \partial G, \Delta u \geqq 0 \text { a.e. }\right\}, \\
W & =\left\{w \in L^{p}(G) ; w \in C(\bar{G}), w \geqq 0\right\},
\end{aligned}
$$

we obtain as a special case of the above theorem that the classic problem $\Delta u=e^{u}$ in $G, u=\phi$ on $\partial G$ has a solution $u$ analytic in $G$.

Example 2. Let

$$
\theta(u) v=\left(1+u_{x}^{2}+u_{y}^{2}\right)^{-3 / 2}\left\{\left(1+u_{y}^{2}\right) v_{x x}-2 v_{x y} u_{x} u_{y}+\left(1+u_{x}^{2}\right) v_{y y}\right\}
$$


for $u, v \in H^{2, p}$, i.e., $\theta(u) u=0$ is the minimal surface equation. With $U, W$ as in (3.2) a very naive calculation yields that there exist constants $k, \gamma$, depending only on $G, p$, such that $k(s)$, defined by (2.4), satisfies

$$
\begin{aligned}
k(s) \geqq k_{1}(s)= & k\left[1+2 \gamma^{2} s^{2}\right]^{-3 / 2} \\
& \cdot\left[1-\gamma^{2} s^{2} \max \left\{2,\left[1+2 \gamma^{2} s^{2}\right]^{3 / 2}\left[1+\gamma^{2} s^{2}\right]^{-3 / 2}\right\}\right] .
\end{aligned}
$$

Application of Theorem 2.1 with $m=\max s k_{1}(s)$ yields

THEOREM 3.2. For any $f \in W$, with $\|f\|_{0, p}+\langle\phi\rangle_{2-(1 / p), p} \leqq m$ the problem $\theta(u) u=f$ in $G, u=\phi$ on $\partial G$ has a solution $u \in C^{2}(G)$.

REMARK 2. We have not assumed that $G$ is convex, but the condition $\langle\phi\rangle_{2-(1 / p), p} \leqq m$ is considerably stronger than the B.S.C. condition of [11], even if $G$ is convex. Even when the region is convex a centrally symmetric example on the unit disk shows that some additional restriction on $f$ is necessary.

EXAMPLE 3. Using the notation of [9], let $G$ be a bounded domain in $E^{n}$, now of class $L_{2+\alpha}, 0<\alpha<1$. Suppose that $\phi \in C_{2+\alpha}$ is given. Put

$$
U=\left\{u \in C_{2+\alpha} ; u=\phi \text { on } \partial G\right\}, \quad W=0 \in C_{\alpha},
$$

and

$$
\theta(u) v=a^{i j}\left(x, u, \frac{\partial u}{\partial x}\right) \frac{\partial^{2} v}{\partial x_{i} \partial x_{j}}+b^{i}\left(x, u, \frac{\partial u}{\partial x}\right) \frac{\partial v}{\partial x_{i}}+c\left(x, u, \frac{\partial u}{\partial x}\right) v
$$

where the summation convention has been used. We make the assumption that the coefficients $a^{i j}, b^{i}, c$, are continuous functions of their arguments, and also that

(i) For each $u \in U$ there exists an $M(u)>0$, depending on $u$, such that

$$
\max \left[\left|a^{i j}\left(x, u, \frac{\partial u}{\partial x}\right)\right|_{\alpha},\left|b^{i}\left(x, u, \frac{\partial u}{\partial x}\right)\right|_{\alpha},\left|c\left(x, u, \frac{\partial u}{\partial x}\right)\right|_{\alpha}\right] \leqq M(u) .
$$

Further

$$
\sup _{|u|_{2+\alpha} \leqq s} M(u)=M(s), \quad u \in U
$$

is finite

(ii) For each $u \in U$ there is a number $m(u)>0$ such that

$$
a^{i j}\left(x, u, \frac{\partial u}{\partial x}\right) \xi_{i} \xi_{j} \geqq m(u) \sum_{i} \xi_{i}^{2} \quad \text { for real } \xi_{1}, \cdots, \xi_{n} .
$$

Further 


$$
\inf _{|u|_{2+\alpha} \leqq s} m(u)=m(s)>0, \quad u \in U .
$$

(iii) For each $u \in U, c(x, u, \partial u / \partial x) \leqq 0$.

From the Schauder boundary estimates we then have for any $u$, $v \in U$, with $\theta(u) v=0, k(s)|v|_{2+\alpha} \leqq|\phi|_{2+\alpha}$ if $|u|_{2+\alpha} \leqq s$ where $k(s)$ $=\inf _{|u| 2+\alpha \S s}[c(u)]^{-1}, u \in U$, and $c(u)$ is the constant normally appearing on the right in the boundary estimates, and depending on $m(s), M(s)$. Using this estimate, Theorem 2.1 now gives

THEOREM 3.3. Under the assumptions (i)-(iii) the equation $\theta(u) u=0$ in $G, u=\phi$ on $\partial G$ has a solution $u \in C_{2+\alpha}$ if there exists an $s_{0}$ such that $s_{0} k\left(s_{0}\right) \geqq|\phi|_{2+\alpha}$.

COROLlaRy. If there exist numbers $m, M>0$ such that (i) $m(s) \geqq m$, (ii) $M(s) \leqq M$ for all $s$, then the Dirichlet problem of the preceding theorem has a solution for all $\phi \in C_{2+\alpha}$.

REMARK 3. The standard exposition of the theory, being based on the Nash-De Giorgi estimates [8], [5] for $n>2$, has for this reason been limited to equations of divergence form. This is not necessary above. The perturbation Theorem 2.2, can of course be applied.

\section{REFERENCES}

1. F. E. Browder, Nonlinear elliptic boundary value problems. II, Bull. Amer. Math. Soc. 69 (1963), 862-874.

2. R. Courant and D. Hilbert, Methods of mathematical physics, Vol. 2, WileyInterscience, New York, 1962.

3. J. Cronin, Fixed points and topological degree in nonlinear analysis, Math. Surveys No. 11, Amer. Math. Soc., Providence, R. I., 1964.

4. G. F. D. Duff, Eigenvalues and maximal domains for quasi-linear elliptic equation, Math. Ann. 131 (1956), 28-37.

5. E. De Giorgi, Sulla differenziabilitd e l'analiticitd delle estremali degli integrali multipli regolari, Mem. Accad. Sci. Torino (3) 3 (1957), 25-43.

6. M. D. George, Completely well-posed problems for nonlinear differential equations, Proc. Amer. Math. Soc. 15 (1964), 96-100.

7. N. Levinson, Dirichlet problem for $\Delta u=f(p, u)$, J. Math. Mech. 12 (1963), 567-576.

8. J. Nash, Continuity of solutions of parabolic and elliptic equations, Amer. J. Math. 80 (1958), 931-954.

9. L. Nirenberg, Estimates and existence of solutions of elliptic equations, Comm. Pure Appl. Math. 9 (1956), 509-529.

10. M. Schechter, Some $L^{p}$ estimates for partial differential equations, Bull. Amer. Math. Soc. 68 (1962), 470-474.

11. G. Stampacchia, On some regular multiple integral problems in the calculus of variations, Comm. Pure Appl. Math. 16 (1963), 383-421.

University of California, Los Angeles 